\title{
Presentation of an Estimator for the Hurst Parameter for a Self- Similar Process Representing the Traffic in IEEE 802.3 Networks
}

\author{
Ginno Millán, Gastón Lefranc
}

Pontificia Universidad Católica de Valparaíso

Escuela de Ingeniería Eléctrica

Avda. Brasil \#2147. Valparaíso - Chile

E-mail: ginno.millan@gmail.com and glefranc@ucv.cl

\begin{abstract}
The hypothesis for the existence of a process with long term memory structure, that represents the independence between the degree of randomness of the traffic generated by the sources and the pattern of traffic stream exhibited by the network is presented, discussed and developed. This methodology is offered as a new and alternative way of approaching the estimation of performance and the design of computer networks ruled by the standard IEEE 802.3-2005.
\end{abstract}

Keywords: Computer networks, IEEE 802.3-2005 standard, network traffic, selfsimilar process.

\section{Introduction}

The positioning and consolidation of Ethernet as a predominant standard in the local and extensive coverage computer network field against some traditional technologies, such as Frame Relay, DQDB and ATM, are facts that are explained by its main characteristics, i.e. compatibility and interoperability among some Ethernet equipment from different speeds, high performance, scalability and self-configuration capacity, independence IP addressing and, undoubtedly, the usual scale economy.

Ethernet, initially at $3 \mathrm{Mb} / \mathrm{s}$, has evolved from $10 \mathrm{Mb} / \mathrm{s}$ to $10 \mathrm{~Gb} / \mathrm{s}$ in the last twenty-two years. The latter does not consider the IEEE 802.3ba standard, which specifies the Ethernet at 40Gb/s and 100 $\mathrm{Gb} / \mathrm{s}$ since 2007. Furthermore, it has also evolved from using simple bridges developed for the network bridging with identical physical protocols and average access to $\mathrm{N} * 10 \mathrm{~Gb} / \mathrm{s}$ switches [1], [2]

Two very interesting, striking and critical aspects are involved in this continuous evolution underwent by the Ethernet networks. The first is related to the complete abandonment of the origin of the halfduplex shared system, which has yielded to full-duplex links. On the other hand, the second involves its extension, as Ethernet has evolved from LAN range distances to WAN range coverages [3]. Furthermore, although both changes have gradually occurred, they result quite critical from the Ethernet point of view, as both represent the disappearance of the mechanism controlling the media access regulated by the CSMS/CD protocol and also assume a strong and radical change in the transmission media, which tends to the massive and total use of the optical fiber to support the ever demanding application of enormous bandwidths.

In general, the local area networks, particularly Ethernet, were developed as high capacity interconnected networks; in contrast to WAN network technologies that are based on switching and transmission flow generally lower than those available in the LAN networks. However, the evolution of the different technologies used in both environments now converges in solutions based in Ethernet and its different specifications. Therefore, the current Ethernet networks are switched and almost totally made from fullduplex links, but they also incorporate the multiplexing according to the IEEE 802.1Q standard and bear transmission distances similar to those involved in the conventional WAN links. This degree of evolution 
is largely ascribed to great development achieved by the Ethernet switches, as there has not only been an increase in the operational transparency and simplicity degree but also a direct effect in the incorporation of additional functionality to the switching, which, from the standard point of view, involves the extension of the format of the original frame and the incorporation of labeling for VLAN and the priority establishment for service classes, the size increase of the CSMA/CD carrier signal, the incorporation of burstiness packets to try to compensate the network velocity loss caused by the extension bits of the carrier and, most of all, in the complete abandonment of the restraint and solution conflict mechanism given by the exponential backward algorithm [4], [5].

On the other hand, the migratory tendency towards Ethernet networks without shared media is confirmed through the incorporation of the IEEE 802.1X, IEEE 802.1w standards (RSTP currently included in the IEEE 802.1D standard) and the IEEE 802.1s standard (MSTP; currently included in the IEEE 802.1Q standard). These establish the dedicated full-duplex links as an essential requirement for the correct operation. It is important to point out these dedicated links are not only necessary to obtain the best benefits from the network, but also to ensure the logical link control (LLC), simplifying the protocols and making possible the rapid convergence mechanisms in layer two.

One example of the latter is the IEEE 802.3e standard (Ethernet at $10 \mathrm{~Gb} / \mathrm{s}$ ), which does not involve the use of half duplex links in the specifications as the IEEE $802.3 \mathrm{z}$ (Ethernet at $1000 \mathrm{Mb} / \mathrm{s}$ ). The use in the IEEE $802.3 \mathrm{z}$ is strictly related to the compatibility with the equipment database previously installed and its aim is to work as a platform in the migration or technological transition processes. It is important to remember that the IEEE $802.3 \mathrm{z}$ is the latest specification in the IEEE 802.3 standard giving native support to this device communication method.

It seems quite interesting the diffusion and flooding dominated in the traditional Ethernet networks as the basic and valid mechanisms to establish the presence or absence of stations; nowadays, however, a minimum diffusion of the frames is sought due to the performance degradation and the exhaustive control of the traffic flow, the same reasons that must be avoided in the WAN networks.

New technologies are similarly incorporated to the large covering networks, which are typical of the LAN environments due to their robustness and great price/assistance relation. These are well established in access and metropolitan environments and, increasingly, in WAN environments [6].

All these arguments guarantee a reassessment of the study of the benefits of the CSMA/CD access control mechanisms, in terms of the impacts of the performance in the current commuted environments, i.e. considering the performance as the useful information quantity that the network is able to transport in relation to the real quantity of transported bits, as well as a characterization of the nature of the traffic under study in terms of a performance pattern capable of describing the temporal evolution as well as the implications on the previously defined performance.

The performance parameter is considered as an active form of measuring the benefits from a network, as this is one of the crucial aspects in the global analysis of the communication systems, considering its impacts in the final users. On the other hand, the traffic characterization among the networks is considered, because the performance observed in the performance parameter depends on it, thus becoming a key factor for the characterization.

Furthermore, a new approach to carry out the modeling processes of the Ethernet networks is justified, as in terms of the evolution previously stated, it is inferred that the natural successor of the IEEE 802.3u standard (Fast Ethernet) must be the IEEE 802.3z standard (Gigabit Ethernet), the latter will give way to the access and WAN environments, the IEEE 802.3ae (Ethernet at $10 \mathrm{~Gb} / \mathrm{s}$ ) and IEEE 802.3ba (Ethernet at $40 \mathrm{~Gb} / \mathrm{s}$ and $100 \mathrm{~Gb} / \mathrm{s}$ ) standards, respectively. The impacts associated to these technological migrations must be adapted and properly evaluated, sized up and classified in terms of the impacts on the installed equipment databases. This must be previously defined before any adoption.

However, the existence of self-similar traffic patterns is accepted on the empirical fact that these are characterized by the constant presence of package traffic bursts through different time scales and that the characteristic property of the self-similar processes is the Long Range Dependence (LRD), which 
is observed when the trunking level increases [7]. Then, the fractal performance of this type of traffic does not coincide with the performance traditionally modeled through the Poisson processes, which are characterized by the absence of bursts and a low variability that is reflected in the temporal independence between the samples. In short, these are processes that show a short-term temporal dependence rejecting the relation between temporarily distant processes, i.e. these are null memory processes that, therefore, do not considered as valid the presence of any pattern representing the sent traffic.

Then, considering the same arguments and the fact the self-similar phenomena show the same aspect or performance when visualized with different enlargement degrees or different scales of a certain dimension, and also that the temporal series showing self-similarity with respect to time are the object of interest in computing networks, a degree of self- similarity must be established for such series, which must be expressed using a parameter representing the de-growth speed of the self-correlation function. This responds to the fact that a time series is self-similar when the aggregated series involves the same self-correlation function as the original series. The latter is achieved using the Hurst parameter, $\mathrm{H}$, which can be estimated through different methods, being the Whittle the one with the highest statistical rigor.

The Whittle estimator calculation may be carried out from different algorithms, all of which need the underlying stochastic process. In this paper a variation of this estimator is proposed, which allows the obtaining of the self-similarity degree with an acceptable commitment relation between the cost of the computing model (higher inconvenient when obtaining the Whittle estimator no matter the chosen method) and the estimation quality.

This proposal formally involves the modification of the Whittle local estimator, or semi-parametric Gaussian estimator, of the memory parameter in the short-term standard process stated in [8]. Then, all the advantages of the original technique are expected, which shows all its main attributes as an alternative to the regression technique of the periodogram logarithm shown in [9]. It is expected that under less restrictive suppositions, an asymptotic efficiency profit form is shown. Therefore, an analysis of the asymptotic performance of the original semi-parametric Gaussian estimator must be carried out on the memory parameter in processes with cyclic or seasonal memory, thus allowing divergences or spectral zeros of asymmetric type. Then, modifying the original algorithm, the consistency and asymptotic normality needed for the characterization of the traffic flows will be obtained.

\section{Problem Definition}

The traffic analysis based on the queuing theory has resulted in great help to the network design and the system analysis when carrying out capacity planning and performance prediction [10]. However, there are some cases in the real world in which all predicted results from a queuing analysis significantly differ from the performance observed in reality [11]. In this sense, it must be remembered the validity of the analysis based on the queuing theory depends on the Poisson nature of the data traffic, and when dealing with Poisson processes, the representation of the length of each arrival and the time between each frame arrival are represented by independent and exponentially arranged random variables. Therefore, these are null memory models. Thus, being this the case, of models in those that the probability of an arrival in an instant, is independent of the instants of previous arrivals, property that is not completed in the nets of commutation of packages. However, it is acknowledged the objective of these suppositions correspond to relatively simple models, from an analytical point of view.

According to the results obtained in [12], different authors have studied the existence of a temporal dependence and the establishment of the great impact involved in the assistance of a queuing system outstands. Therefore, there is plenty of literature on entry traffic models showing more or less complex correlation structures, which are used in cases when the telecomputing system model under study allows an adequate analytical treatment. In any case, all these models, mainly of Markovian nature, reject the correlation from a given temporal separation, even when it may be arbitrarily increased at the expense of making the model complicated with additional parameters. 
In [13] and [14] is shown that after several measurements on an Ethernet network, the traffic shows a self-similar or fractal nature (understanding traffic as the frame quantity in the network by a time unit). This becomes apparent with the existence of a long-term correlation.

The self-similar characteristic of the traffic in WAN networks is shown in [15] and [16]. Furthermore, the fractal nature of the data flow from the protocols involved in the signaling system 7 (SS7) in the common channel signaling networks is shown in [17].

The self-similar nature of the traffic due to the WWW is shown in [18], using the experimental evidence, the possible causes and origin are shown.

The self-similar nature in the traffic of the variable bit rate (VBR) is shown in [19] and then in [20].

In [21] is shown that the probability distribution that is followed by the queuing size of a multiplexor shows a Weibull-type asymptotic fall when using certain self-similar process as the entry traffic. Then, in [22] it is shown this fall is even slower and hyperbolic when other self-similar processes are used.

Reference [23] shows the effective band width estimated through Markov models, where the queue size distribution show an exponential fall, highly underestimates the loss rate of cells in different orders of magnitudes in ATM networks.

An outstanding long-term positive correlation in the added traffic is shown in [24], from the analysis of a voice and data multiplexer, where some high delays were obtained, higher than those obtained using the Poisson models.

In reference [25] the number of entries is shown, counted in adjacent time intervals that result in the superposition of multiple independent and homogeneous voice sources susceptible to be treated through updating process models. This is a complex process that includes strong correlations and involves a significant impact in the assistance of the studied system.

The interesting thing about this and other studies is that they highlight the impact the long-term temporal dependence have on the assistance of the communication networks, which is intrinsic to the most diverse traffic types, in contrast to other models that do not include this due to analytical simplicity (renewal processes, for example) or that show a more analytically complex correlation structure (such as the Markov or generally self-regressive models). All these are known as short range dependence models (SRD). Furthermore, the main problem these traditional models involve is that they require a great number of parameters to characterize the strong correlations existing among different traffic types in a network. In this study, it is clearly stated that while the parameter number increases, the analytical complexity also increases (not necessarily in a lineal form) and that there is also a difficulty to give a physical interpretation to all the parameters and to estimate them from empirical data.

The self-similarity and fractability characteristics help to describe a phenomenon where certain object property is preserved in relation to the temporal and spatial scalability and in a self-similar and fractal object. Thus, the magnified parts are similar to the form of the complete object, where the similarity is measured in some proper form. Then, the simpler form of self-similarity is obtained by reconstruction through the iteration of a certain procedure. If this process is indefinitely repeated for each new segment, any portion of the object can be magnified to exactly reproduce a bigger portion, no matter how small the portion. This property is known as "exact self-similarity" [26]. No exact self- similar characteristics are intended to be observed in a highly random process such as the one involving the packets arrival to a data network; however, the traffic observed as sample traces from a stochastic process are considered and the similarity is only restricted to certain specific statistics of the temporal series though adjusted in scale. Furthermore, the exact self-similarity in abstract mathematical objects and approximate self-similarity for each considered specific execution are also discovered. No exact self-similarity is expected from the network traffic under study; however, a self-similar stochastic behavior is expected and, according to this, the second order statistics may be used to capture the process variability in order to determine the self-similarity rate. In fact, the scale invariance may be defined in terms of the self-correlation function, as the polynomial decrease of this function (in place of the exponential) represents a long-term dependence manifestation similar to the self-similarity, and constitutes the view from which all self-similar 
processes must be interpreted through the development of this investigation.

On the other hand, in relation to the fundamental problem of the self-similar process analysis, or more precisely, to the temporal series showing LRD, i.e. the estimation of the Hurst parameter $(\mathrm{H})$, the methods involved in the literature can be classified in the following two groups:

1. Geographical methods of lineal regression. Used to estimate a statistic $T(x)$ behaving asymptotically for a determined group of $x$ values and, therefore, are based in obtaining the straight line that better adjusts (for that group of $x$ values) to the $\log (T(x))$ against the $\log (x)$ using the least square lineal regression, thus obtaining the $\mathrm{H}$ parameter value directly from the gradient value of that particular straight line.

2. Methods based on Maximum Likelihood Estimators (MLE) for $\mathrm{H}$. These help to minimize the differences between the periodogram of the concerning series and its theoretical spectrum.

The methods involved in the first group are relatively simple and algorithmically fast to implement. However, their main disadvantage is that an asymptotic performance must be first estimated from a finite sample number, which makes the estimation of the $\mathrm{H}$ parameter directly and considerably dependant from the right selection of the $x$ value group. Therefore, the graphic representations result crucial for the confirmation of correspondence between the $x$ values and the lineal performance area and the proper adjustment of the straight line for the represented points. Furthermore, it is important to point out these methods only allow a precise estimation of $\mathrm{H}$, as the resulting confidence intervals involves a high cost for the computing resource and long processing periods, both as a result of the use of the intensive graphic-type methods. The MLE-based methods, on the other hand, though more complex and a higher computing cost, result more flexible and efficient from the statistical inference point of view, as they allow the obtaining of confidence intervals for the $\mathrm{H}$ estimated values. Therefore, these are the most common methods.

This investigation will deal with MLE-based methods to solve the confidence intervals directed to obtain the first approximations for an $\mathrm{H}$ value, which will be then adjusted using the proposed method. In any case, the methods from the first group will be thoroughly analyzed and the results will be contrasted with those resulting from the MLE and the adjustment suggested by the proposed method. Then, and as the analysis starts using MLE, it is important to stress these are methods designed to minimize the differences between the periodogram of the series and the parametric model suggested for the theoretical spectral density. Besides, the exact estimation of the MLE is quite expensive form the computing point of view, so likelihood Gaussian functions are generally used (Gaussian MLE). However, even when these kind of functions are considered, the computing costs results quite high, thus the approximations based on Gaussian MLE are generally used and the most widely used approximation is the Whittle approximation; therefore, the suggested method is based on the modification of one of the variables for the result.

\section{Justification and Proposition of an Efficient Whittle Estimator}

Being $f(\lambda, \theta)$ the parametric form of the spectral density of a Gaussian stationary process, $X_{t}$ where, $\theta=\left(\theta_{1}, \ldots, \theta_{M}\right)$ is the parameter vector to estimate. Then, being $I(\lambda)$ the periodogram of samples defined by

$$
I(\lambda)=\frac{1}{2 \pi N}\left|\sum_{t=1}^{N} X_{t} e^{j t \lambda}\right|^{2}
$$

The approximated MLE o Whittle is the vector

$$
\widehat{\theta}=\left(\widehat{\theta}_{1}, \ldots, \widehat{\theta}_{M}\right)
$$


minimizing the function

$$
\mathrm{Q}(\theta) \widehat{=} \frac{1}{2 \pi}\left\{\int_{-\pi}^{\pi} \frac{\mathrm{I}(\lambda)}{\mathrm{f}(\lambda, \theta)} \mathrm{d} \lambda+\int_{-\pi}^{\pi} \log [\mathrm{f}(\lambda, \theta)] \mathrm{d} \lambda\right\}
$$

in practice, the estimation of the Whittle estimator is done choosing an adequate scale parameter $\theta_{1}$, complying with

$$
f(\lambda, \theta)=\theta_{1} f\left(\lambda, \theta^{*}\right)=\theta_{1} f^{*}(\lambda, \eta)
$$

thus annulling the second addend of (3), i.e.

$$
\int_{-\pi}^{\pi} \log \left[f\left(\lambda, \theta^{*}\right)\right] \mathrm{d} \lambda=\int_{-\pi}^{\pi} \log \left[f^{*}(\lambda, \eta)\right] \mathrm{d} \lambda=0
$$

where $\eta=\left(\theta_{1}, \theta_{2}, \ldots, \theta_{M}\right)$ y $\theta^{*}=(1, \eta)$

In [27] is shown the scale parameter is given by $\theta_{1}=\frac{\sigma_{\varepsilon}^{2}}{2 \pi}$, being the mean-square prediction medium (MSPE).

On the other hand, the discreet version of the Whittle estimator is suggested in [28], which is approximated to 3 using a Riemann addition in the frequency range given by $\lambda_{k}=2 \pi \mathrm{N}^{-1} k$, with $k=1,2, \ldots N^{*}$ (being $N^{*}$ the integer part of $(N-1) / 2$ ). Then, the function to be minimized is given by the expression

$$
\widetilde{Q}\left(\theta_{1}, H\right)=\frac{4 \pi}{N}\left\{\sum_{k=1}^{N^{*}} \frac{I\left(\lambda_{k}\right)}{f\left(\lambda_{k}, \theta_{1}, H\right)}+\log \left[f\left(\lambda_{k}, \theta_{1}, H\right)\right]\right\}
$$

The estimated $\mathrm{H}$ parameter is obtained through the selection of the adequate scale parameter, $\hat{\mathrm{H}}$, a value that minimizes the following expression

$$
\widetilde{Q}^{*}(H)=\widetilde{Q}(1, H)=\sum_{k=1}^{N^{*}} \frac{I\left(\lambda_{k}\right)}{f\left(\lambda_{k}, 1, H\right)}=\sum_{k=1}^{N^{*}} \frac{I\left(\lambda_{k}\right)}{f^{*}\left(\lambda_{k}, H\right)}
$$

where it is verified that

$$
f^{*}(\lambda, H)=\frac{1}{\theta_{1}} f\left(\lambda, \theta_{1}, H\right)=\frac{2 \pi}{\sigma_{\varepsilon}^{2}}
$$

The following disadvantages of the Whittle estimator stand out in its conventional forms, from this point of view:

1. Determination of the parametric form of the spectral density.

2. High estimation period due to graphic method use.

The Central Limit Theorem for self-similar processes result quite useful from the perspective of the application of the Whittle estimator to processes where it is not possible to assure anything in relation to the spectral density, as it is a good approximation for non-Gaussian series, which allow the application of aggregated series to any result from the pure self-similar Gaussian processes, as shown in [29]. Then, it seems quite interesting this theorem allows the supposition that for a temporal series of $\mathrm{N}$ size, whose self-correlation shows a LRD hyperbolic fall, if $\mathrm{m}$ and $\mathrm{N} / \mathrm{m}$ are sufficiently big and the variance is finite, then the FGN process is a good approximation for the aggregated sequences of the series, even when it does not represent a Gaussian approximation [30].

The latter is the base for the variant of the Whittle estimator known as the added Whittle estimator, which gives a more robust and less biased form of the Whittle estimator when no information on the 
exact parametric form of the spectral density is available. In other words, a shorter representative series is obtained given by the expression

$$
X_{k}^{(m)}=\frac{1}{m} \sum_{i=k m}^{(k+1) m-1} X_{i} \quad 0 \leq k \leq[N / m]
$$

And then the Whittle estimator is used, considering the Fractionary Gaussian Noise (FGN) as the parametric model of its spectral density. However, in spite of the fact of using a shorter series considerably reduces the computing cost, the observed problem is that the variance of the estimator increases and so does the self-similarity degree, thus reducing the pattern representativeness degree.

Another problem associated to the method is the impossibility to a priori know the value of the appropriate $\mathrm{m}$. However, in this last sense, a method to represent the estimation of the $\mathrm{H}$ parameter are shown in [31], which were obtained for different $m$ values, and finding a region where the graphics are approximately plain.

The local Whittle estimator is shown in [32], which in contrast to the Whittle estimator, represents a semi-parametric estimator that only specifies the parametric form of the spectral density for those frequencies close to zero, i.e.,

$$
f(\lambda) \sim G|\lambda|^{1-2 H}
$$

when $\lambda \rightarrow 0$

When replacing $f(\lambda, H)$ given by (10) in (2), and integrating up to the $\frac{2 \pi M}{N}$ frequency, with $\frac{1}{M}+\frac{M}{N} \rightarrow$ 0 when $\mathrm{N} \rightarrow \infty$, the following is obtained

$$
Q(G, H) \widehat{=} \frac{1}{M} \sum_{j=1}^{M}\left[\frac{I\left(\lambda_{j}\right)}{G \lambda_{j}^{1-2 H}}+\log \left(G \lambda_{j}^{1-2 H}\right)\right]
$$

When replacing the $\mathrm{G}$ constant by its estimation, given by

$$
\widehat{\mathrm{G}}=\frac{1}{M} \sum_{j=1}^{M} \frac{\mathrm{I}\left(\lambda_{j}\right)}{\lambda_{j}^{1-2 \mathrm{H}}}
$$

the function to be minimized is obtained, i.e.,

$$
\begin{gathered}
\mathrm{R}(\mathrm{H}) \widehat{=} \mathrm{Q}(\widehat{\mathrm{G}}, \mathrm{H})-1 \\
\mathrm{R}(\mathrm{H}) \widehat{=} \log \left[\frac{1}{M} \sum_{j=1}^{M} \frac{\mathrm{I}\left(\lambda_{j}\right)}{\lambda_{j}^{1-2 \mathrm{H}}}\right]-(2 \mathrm{H}-1) \frac{1}{M} \sum_{j=1}^{M} \log \left(\lambda_{j}\right)
\end{gathered}
$$

Nevertheless, the problem still remains, as the selection of the $M$ value results critical and from this value the bias and variance depend, and yet again the bias and variance problem shows. However, as long as $\mathrm{M}$ increases, the estimated value for $\mathrm{H}$ will quickly converge to the real $\mathrm{H}$ value but the spectrum form will continuously fall apart from (6) and the SRD effects will be even greater, thus increasing the bias. Then, as in the previous method, one must choose to represent the estimated value of $\mathrm{H}$ against $\mathrm{m}$ and find the plain region of the graphic. As it has been observed from the methods about the Whittle estimator, all of them require the minimization of an expression, (6) or (14). The most obvious way of carrying out such minimizations is to assess these expressions for a certain number of equidistant $\mathrm{H}$ values $(q)$, which will depend on the selected resolution. However, it must be pointed out that as lightly higher number of samples are needed for the algorithm to be immediately translated into a very high computing cost. 
Then, a reduction through an algorithm is suggested to reduce the computing cost, which is aimed to decrease the number of points to be assessed. Therefore, the convex characteristic of the function in all the domain $[0.5,1$ ( ( will be considered also that the minimal is then unique. Then, a searching method by bi-section used on the function derivative will have to allow the number of evaluated points to be around $\log 2(q)$, which will be of advantage in a significant sample saving, and at the same time it does not settle in relation to the bias and variance commitment.

Therefore, considering the assessment of the derivative in a $\mathrm{H}_{i}$ point may be approximated by a difference coefficient for a sufficiently small increase, h, i.e.,

$$
\mathrm{Q}^{\prime}\left(\mathrm{H}_{\mathrm{i}}\right) \approx \frac{\mathrm{Q}\left(\mathrm{H}_{\mathrm{i}}+\mathrm{h}\right)-\mathrm{Q}\left(\mathrm{H}_{\mathrm{i}}\right)}{\mathrm{h}}
$$

for $h \rightarrow 0$

It is derived the main work hypothesis is related with the establishment of a self-similarity degree based on the Whittle estimator; however, due to the complexity involved in the bias and variance commitment behind all models, it is then suggested that in a reduced point spectrum it is possible to give an answer with an acceptable level of commitment. Therefore, in order to obtain confidence intervals, and taking advantage of the convexity of the function to minimize in the whole dominium $[0.5,1$ (, which implies the presence of a unique minimum, it will be considered that when estimating a unique $\mathrm{H}$ parameter, if $\widehat{H}$ represents the value minimizing the $Q(H)$ function and that $H_{0}$ represents its real value, then

$$
\left(\widehat{\mathrm{H}}-\mathrm{H}_{0}\right) \rightarrow \mathrm{N}\left(\mathrm{O}, \sigma_{\mathrm{H}}\right)
$$

where the $\sigma_{\mathrm{H}}$ parameter will be defined by:

$$
\sigma_{H}^{2}=\frac{4 \pi}{N}\left\{\int_{-\pi}^{\pi}\left[\frac{\partial \log [f(\lambda, H)]}{\partial H}\right]^{2} d \lambda\right\}_{H=H_{0}}^{-1}
$$

Then, the estimation of the derivative given by (14) may also approximate through a difference coefficient for a sufficiently small increase, $h$. However, in this case, the chosen value for $\theta_{1}$ different, because a cancellation is produced. Then,

$$
\sigma_{H}^{2}=\left\{\sum_{k=1}^{N^{*}}\left[\frac{\partial \log \left[f\left(\lambda_{k}, \theta_{1}, H\right)\right]}{\partial H}\right]^{2}\right\}_{H=\widehat{H}}^{-1}
$$

Where the [33] it is suggested in the end that

$$
\sigma_{H}^{2}=\left\{\sum_{k=1}^{N^{*}}\left[\frac{\log \left[f\left(\lambda_{k}, \theta_{1}, \widehat{H}+h\right)\right]}{\partial H}-\frac{\log \left[f\left(\lambda_{k}, \theta_{1}, \widehat{H}\right)\right]}{\partial H}\right]^{2}\right\}^{-1}
$$

\section{Conclusions}

The hypothesis on the existence of a process with a temporary structure of long-term memory was presented, discussed and developed, which was representative of the independence between the randomness degree of the traffic generated by the sources and the pattern of the traffic flow shown by the network. The latter has been shown to be considered as a new form and alternative to cover the performance and network design estimation topics that are ruled by the IEEE 802.3-2005 standard.

The traditional models based on the Poisson processes, or even more general, based on short-term dependency processes are unable to describe the performance of he current data networks, particularly 
those related to the switched Ethernet networks according to the IEEE 802.3-2005 standard. Consequently, it is necessary to redefine the study of the load systems, considering self-similar entry processes as a result of the self-similar traffic demand imposing new requirements in the network design, especially in what the buffering strategies are concerned. All methods traditionally used to assess the Whittle estimator show the disadvantages of the need to know the parametric form of the spectral density, as well as a high computing cost resulting from the intensive use of graphic methods. It is estimated these problems may be solved as far as it is feasible to introduce an algorithm that reduces the number of points to be assessed. This does not only mean reduced costs in computing processing, but also a new alternative to be considered in the study of the self-similar or fractal traffic consideration on the benefits of a network.

A convex function to carry out the minimization of the generalized function of the local Whittle estimator is suggested for a delimited domain, which involves the advantage to include a sole minimum that is completely individualizable, and thus using a bi-sectional searching method applied on the derivative of the function, should allow the determination of a point around which all values fluctuate. Therefore, this allows the availability of a plain region in which the value of the $\mathrm{H}$ parameter is perfectly approximated by a difference coefficient. The latter must be translated into savings in the computing costs and processing time that endorse the new proposed model.

The development of the simulations is being tackled through literature search as a first stage, which must clearly include the simulation techniques and statistical analysis for long-term dependency series, as it is considered it is not enough to be able to deduce the results from some aggregations, but there must be a standardization of the procedures to specifically study the long-term dependencies. This area requires urgent attention, as most of the operational costs involved in the computing capacity directly depend on it.

\section{Bibliography}

[1] Metcalfe, M. and Boggs, R. Ethernet: Distributed Packet Switching for Local Computer Networks.Communications of the ACM, Vol. 19 Nº7, 1976.

[2] Ibańez, G. Contribución al Diseńo de Redes de Campus Ethernet Autoconfigurables. Ph.D. thesis, Dept. Ing. Telemática, Universidad Carlos III de Madrid, Madrid, Espańa, 2005.

[3] Ibańez, G. Contribución al Diseńo de Redes de Campus Ethernet Autoconfigurables. Ph.D. thesis, Dept. Ing. Telemática, Universidad Carlos III de Madrid, Madrid, Espańa, 2005.

[4] García, J., Ferrando, S., and Piattini, M., Redes para Proceso distribuido, Madrid, Ra-Ma, pp. 127160, 1997.

[5] Zacker, C., Redes. Manual de Referencia, Madrid, McGraw-Hill, pp. 275-341, 2002.

[6] Halabi, S.,Metro Ethernet. The Definitive Guide to Enterprise and Carrier Metro Ethernet Applications. Indianapolis, Cisco Press, pp. 1, 2003.

[7] Leland, W., Taqqu, M., Willinger, W., and Wilson, D., On the Self-similar Nature of Ethernet Traffic, IEEE/ACM Trans. Networking, vol. 2, no. 1, pp. 1-15, 1994.

[8] Robinson, P., Gaussian Semiparametric Estimation of Long-range Dependence,Annals of Statistics, Vol. 3, no 1995b, pp. 1630-1661, 1983.

[9] Geweke, J., and Porter-Hudak, S., The Estimation and Application of Long Memory Time Series Models. J. Timer Ser. Anal. 4, pp. 221-238, 1983. 
[10] Stallings, W., Internet y Redes de Alta Velocidad. Rendimiento y Calidad de Servicio. 2nd ed., Madrid, Pearson Prentice Hall, pp. 224-225, 2004.

[11] Stallings, W., Internet y Redes de Alta Velocidad. Rendimiento y Calidad de Servicio. 2nd ed., Madrid, Pearson Prentice Hall, pp. 224-225, 2004.

[12] Kleinrock, L., Communication Nets, New York, McGraw-Hill, 1972.

[13] Leland, W., Taqqu, M., Willinger, W., and Wilson, D., On the Self-similar Nature of Ethernet Traffic,Computer Communications Review, Vol. 23, pp. 183-193, 1993.

[14] Leland, W., Taqqu, M., Willinger, W., and Wilson, D., On the Self-similar Nature of Ethernet Traffic, IEEE/ACM Trans. Networking, vol. 2, no. 1, pp. 1-15, 1994.

[15] Klivansky, S., Mukherjee, S., and Song, C., Factor Contributing to Self-similarity over NFSNet, Georgia Institute of Technology, 1995.

[16] Paxon, V., and Wilson, D., Wide-area Traffic: The failure of Poisson Modeling, IEEE/ACM Trans. Networking, Vol. 3, no. 1, pp. 266-244, 1995.

[17] Duffy, D., Mcintosh, A., Rosenstein, M., and Willinger W., Statistical Analysis of CCSN/SS7 Traffic Data from Working CCS Subnetworks, IEEE Journal on Selected Areas in Communications, Vol. 12, pp. 544-551, 1994.

[18] Crovella, M., and Bestavros, A., Self-similarity in World Wide Web Traffic: Evidence and Possible Causes, IEEE/ACM Trans. Networking, Vol. 5, no. 6, pp. 835-846, 1997.

[19] Garret, M., and Willinger, W., Analysis, Modeling and Generation of Self-similar VBR video Traffic, Proc. ACM SIGCOMM'94, pp. 269-280, London, 1994.

[20] Beran, J., Sherman, R., Taqqu, M., and Willinger, W., Long-range Dependence in Variable-bit-rate Video Traffic, IEEE Trans. Communications, Vol. 24, no. 2, pp.1566-1579, 1995.

[21] Norros, I., A Storage Model with Self-similar Input, IEEE Tans. Queueing Systems, Vol. 16, pp. 387-396, 1994.

[22] Likhanov, N., Tsybakov, B., and Georganas, N., Analysis of an ATM Buffer with Self-similar “Fractal”) Input Traffic, Proc.IEEE INFOCOM'95, pp. 985-992, Boston, MA, 1995.

[23] Elwaid, A., and Mitra, D., Effective Bandwidth of General Markovian Traffic Sources and Admission Control of High-speed Networks, IEEE/ACM Trans. Networking, Vol. 1, no. 3, pp. 329-343, 1993.

[24] Sriram, K., and Whitt, W., Characterizing Superposition Arrival Processes in Packet Multiplexers for Voice and Data, IEEE Journal on Selected Areas in Communications, Vol. 4, pp. 833-846, 1986.

[25] Heffes, H., and Lucantoni, D., A Markov Modulated Characterization of Packetized Voice and Data Traffic and Related Statistical Multiplexer Performance, IEEE Journal on Selected Areas in Communications, Vol. 4, no. 6, pp. 856-868, 1986.

[26] Bravo, J., and Marrone, L., Tráfico Autosimilar. Algoritmo Algebraico para Asignación Dinámica del Buffer, Revista INGENIUS, Ed. 2, Facultad de Ingenierías, Universidad Politécnica Salesiana, Ecuador, 2007.

[27] Beran, J., Statistics for Long-memory Processes, in Generalized Additive Models (Monographs on Statistics and Applied probability), New York, Chapman \& Hall, 2000. 
[28] Geweke, J., and Porter-Hudak, S., The Estimation and Application of Long Memory Time Series Models. J. Timer Ser. Anal. 4, pp. 221-238, 1983.

[29] Taqqu,M., and Teverovsky, V., A Practical Guide to Heavy Tails: Statistical Techniques Ans Applications. http://citeseerx.ist.psu.edu, 1983.

[30] Taqqu,M., and Teverovsky, V., A Practical Guide to Heavy Tails: Statistical Techniques Ans Application. http://citeseerx.ist.psu.edu, 1983.

[31] Leland, W., Taqqu, M., Willinger, W., and Wilson, D. (1994), On the Self-similar Nature of Ethernet Traffic, IEEE/ACM Trans. Networking, Vol. 2, no. 1, pp. 1-15.

[32] Robinson, P., Log-periodogram Regression of Time Series with Long-range Dependence, Annals of Statistics, no. 23, pp. 1048-1072, 1995.

[33] Millán, G., and Lefranc, G., Proposición de un Estimador del Parámetro de Hurst para un Proceso Autosimilar Representativo del Grado de Aleatoriedad del Tráfico Registrado en Redes IEEE 802.32005. XVIII Congreso de la Asociación Chilena de Control Automático (ACCA), 2007. 